\title{
ORIGINAL ARTICLE \\ Study on the safe range of shortening of the spinal cord in canine models
}

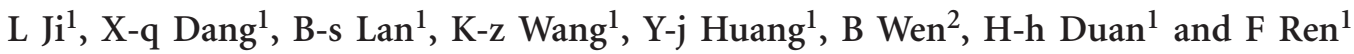

Study design: Experimental dog model of spinal cord shortening.

Objectives: To clarify the relationship between the amount of shortening of the spinal cord and the degree of injury it may induce, and to determine the safe range of the shortening.

Setting: Xi'an Jiaotong University, China.

Methods: Thirty adult dogs were randomly allocated to five groups. Dogs in Group A (sham operation control) underwent spondylectomy to have two-thirds of the thirteenth thoracic segment (T13) resected, without bone-to-bone contact of the adjacent vertebral bodies. Those in Group B, C, D and E had one-third, half, two-thirds and total of their T13 resected, respectively, with boneto-bone contact. Somatosensory-evoked potentials (SEP) and spinal cord blood flow (SCBF) were detected. The histopathologic changes of spinal cord tissue were observed by hematoxylin and eosin stain and electron microscope.

Results: The shortening of the spinal cord $<$ half of a vertebral segment height caused a reversible change of SEP. Whereas, the changes resulted from the shortening of more than two-thirds of a vertebral segment height did not return to the normal level. SCBF increased temporarily when the shortening was within two-thirds of a vertebral segment height; whereas, it decreased progressively when the length of the shortening was equal to one vertebral segment height. More serious hemorrhage occurred as the shortening increased.

Conclusion: Shortening of half of a vertebral segment height will not induce spinal cord injury (SCI), while that between half and two-thirds of a vertebral segment may lead to incomplete SCl.

Spinal Cord (2013) 51, 134-138; doi:10.1038/sc.2012.99; published online 4 September 2012

Keywords: spinal cord injury; spinal cord shortening; vertebrectomy; spinal cord blood flow; somatosensory evoked potential; histopathologic

\section{INTRODUCTION}

Vertebrectomy is commonly performed in treating spinal deformity, tumors of the spinal column and other abnormal spinal conditions. ${ }^{1-3}$ In this process, spinal shortening is a basic and commonly performed operation for the correction of spinal deformity and the fusion of osteotomy surface. ${ }^{2,4,5}$ However, there exists the risk of neurologic complications induced by spinal cord injury (SCI) due to overshortened spinal cord. ${ }^{2}$ These complications can be catastrophic for the patients and are very costly. Therefore, it is of great importance to clarify the pathophysiological changes of spinal cord shortening and determine the safe range of the shortening so as to prevent SCI.

However, the safe range of shortening of the spinal column is still in debate. Two previous studies recognized the association between SCI and the range of spinal column shortening, but reached different conclusions in the safe range. ${ }^{6,7}$ It seems that further research is necessary to confirm the safe shortening range. Besides, we have found no report about the serial changes of somatosensory-evoked potentials (SEP) and spinal cord blood flow (SCBF) over time with different amount of spinal shortening. The present study was therefore conducted, in which a modified animal model was used and detailed variations of SCBF and SEP were quantitatively recorded over a longer period of time at each stage of shortening. The obtained data would be of significance in the design and implementation of therapeutic or prophylactic treatment strategies. To our knowledge, it was for the first time that the histopathologic changes at different stages of spinal shortening were observed by hematoxylin and eosin (HE) stain and electron microscope.

\section{MATERIALS AND METHODS}

\section{Animals}

Thirty healthy adult dogs (19 males and 11 females, Xi'an Jiaotong University, Xi'an, China) with body length ranging from 88 to $98 \mathrm{~cm}$, were used. The dogs were randomly allocated to five groups. Group A was the sham control group, and the dogs underwent a spondylectomy of two-thirds of the vertebral body and the lamina of the thirteenth thoracic segment (T13). Group B, C, D and E were the experimental groups, and the animals had one-third, half, two-thirds and the total of their T13 vertebral bodies and laminas resected, respectively, and then the proximal and distal bone surfaces were contacted with each other

\section{Modeling of the shortening of the spinal cord}

Animals were anesthetized with $2.5 \%$ sodium pentobarbital $(1 \mathrm{mg} / \mathrm{kg})$ by intraperitoneal injection. The mean blood pressure was measured through a cannula inserted into the carotid artery. The animals were ventilated mechanically with air, and vesicostomy was performed. Proper amount of T13 lamina was removed. Two pedicle screws (diameter: $3.5 \mathrm{~mm}$, length:

${ }^{1}$ Department of Orthopedic Surgery, The Second Affiliated Hospital, School of Medicine, Xi'an Jiaotong University, Xi'an, China and ${ }^{2}$ Department of Orthopedic Surgery, The Hardware Industry 521 Hospital, Xi'an, China

Correspondence: Professor B-s Lan, Department of Orthopedic Surgery, The Second Affiliated Hospital, School of Medicine, Xi'an Jiaotong University, Xi'an 710004, China. E-mail: LBS526@126.com

Received 5 April 2012; revised 31 July 2012; accepted 3 August 2012; published online 4 September 2012 
$3.2 \mathrm{~cm}$ ) were inserted parallel in T12 and L1, and were fixed using a titanium rod that had been bent in advance to fit the normal kyphosis. Then vertebrectomy and laminectomy of T13 were performed. The spinal cord and nerve roots were cautiously protected in the operation. Finally the proximal and distal bone surfaces were gradually contacted and fixed by prelum instrument ${ }^{7,8}$ (Figure 1). Principles of laboratory animal care were followed and all procedures were conducted according to the guidelines established by the National Institutes of Health.

\section{Monitoring of SEP}

SEP values were recorded by an electromyographic evoked potential machine (NHK30-Medelec Synergy, Oxford, UK) at the following time points: after anesthesia but before surgery, after laminectomy, after vertebrectomy but before the spinal cord shortened, and then 5, 15, 30, 60, 120 and 360 min after the spinal cord shortened. The tibial nerves were stimulated by square-wave electrical stimuli of $0.2 \mathrm{~ms}$ duration delivered with a frequency of $1 \mathrm{~Hz}$ using a bipolar stimulation electrode. The stimulus intensity was $11 \mathrm{~Hz}$. The active electrode was placed halfway between nasion and inion at the midline of the scalp $(\mathrm{Cz}),{ }^{9}$ and the reference electrode was at the left ear $\left(\mathrm{A}_{1}\right),{ }^{9}$ and the ground electrode was at the iliac crest contralateral to the stimulation site. ${ }^{10}$ The peak latency and the peak-to-peak amplitude of the N2-P2 complex were analyzed. ${ }^{10}$

\section{Monitoring of SCBF}

SCBF was recorded at the following time points: after laminectomy (this was as the baseline value), after vertebrectomy but before the spinal cord shortened, and then 5, 15, 30, 60, 120 and $360 \mathrm{~min}$ after the spinal cord shortened. Laser doppler flowmetry technique (Periflux system 5000 with the Pf403 probe; Perimed, Stockholm, Sweden) was used, with a wave length of $650 \mathrm{~nm}$. The probe was placed on the dorsal surface of the spinal $\operatorname{cord}^{11}$ and fixed to the spinous process in order to avoid the motion of the spinal cord relative to the probe caused by breathing. The value was expressed as a percentage relative to the baseline level. ${ }^{12}$

\section{Histopathological examination (HE staining and electron microscope)}

Six hours after surgery, the animals were anesthetized with a lethal dose of pentobarbital and perfused with phosphate-buffered saline followed by $4 \%$ paraformaldehyde fixative. A $1.5-\mathrm{cm}$ cord segment centered on the maximum kinking of the dura, or a segment centered on the midpoint of the exposed dura when the morphous of dura kept normal, was removed and fixed overnight at $4{ }^{\circ} \mathrm{C}$.

The rostral half of the sample tissue was embedded in paraffin, sectioned transversely at a thickness of $5 \mu \mathrm{m}$, and stained with HE for histopathological examination at the light microscopic level.
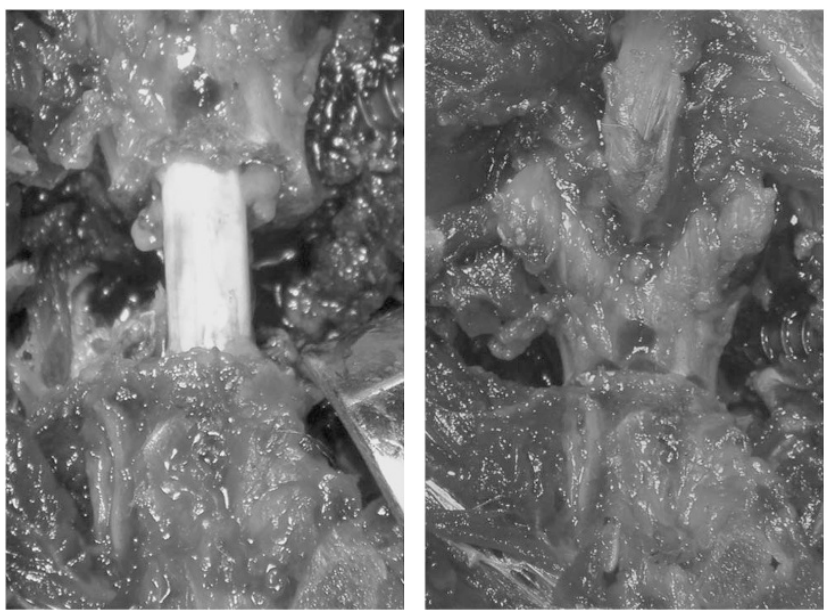

Figure 1 Vertebrectomy of T13 and contacting of the proximate and distal cross sections.
The distal half of the sample tissue was processed for electron microscopy. The tissue was fixed in $2.5 \%$ glutaraldehyde and then post-fixated in $1 \%$ osmium tetroxide, after which it was embedded in 618 epoxide resin. Finally, $100 \mathrm{~nm}$-thick tissue sections were mounted on 100 mesh copper grids and examined with a Hitachi H600 electron microscope (Hitachi, Tokyo, Japan).

\section{Statistical analysis}

The study was designed as repeated measures on two factors at multiple levels. Latency, amplitude and percentage of SCBF relative to the baseline were expressed as mean \pm s.e.m. and analyzed using SPSS 13.0 for Windows (SPSS Inc., Chicago, IL, USA). Significance was set at $P<0.05$.

\section{RESULTS}

SEP

The latencies and amplitudes showed no significant change after laminectomy. In Group D and Group E, the peak-to-peak amplitudes decreased significantly $(P=0.036)$, but returned to the normal level soon $(P=0.310)$. The latency showed a temporary increase $(P=0.019)$ in Group E, and also returned to the normal level in a short period.

The latencies of all the experimental groups increased and the amplitudes of these groups decreased significantly at $5 \mathrm{~min}$ after the shortening of the spinal cord. For Group B and C, the changes returned to the normal level in $6 \mathrm{~h}$ after surgery. However, when the length of the shortening was two-thirds (Group D) or the total (Group E) of a vertebral segment height, the latencies remained significantly prolonged (Figure 2, Table 1) and the amplitudes remained significantly lower (Figure 3, Table 2) until the end of the survival period. The mean amplitude of Group $\mathrm{E}$ presented a progressive decline instead of a tendency to return. Two of the amplitudes declined over 50\% and two disappeared finally.

\section{SCBF}

The SCBF of Group B, C and D increased significantly at 5 min after the shortening of the spinal cord, it then showed a downward tendency. The SCBF in group B returned to the baseline level in $2 \mathrm{~h}$ $(P=0.191)$, the one in group $\mathrm{C}$ was a little higher than the baseline

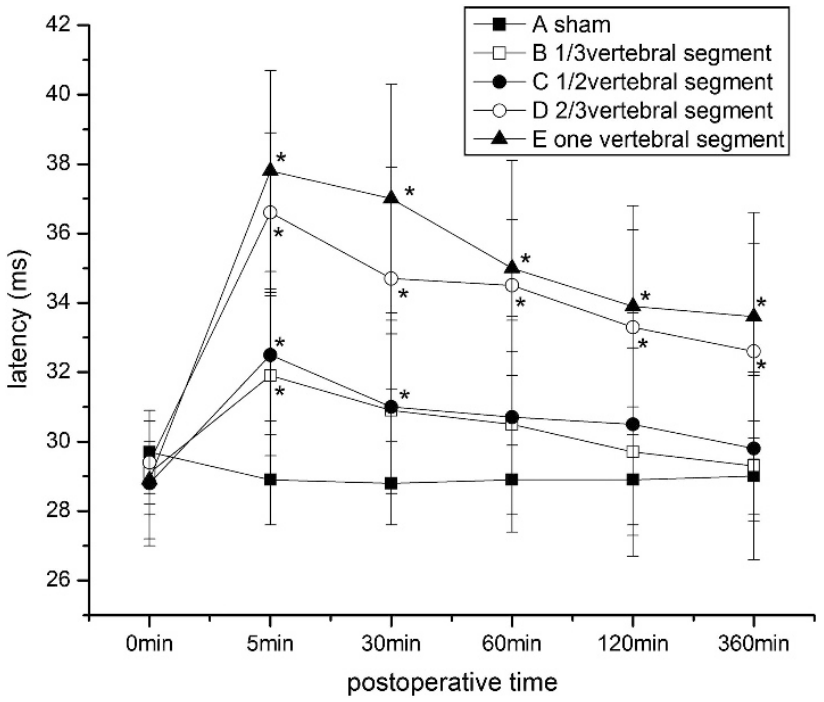

Figure 2 Changes of the latency of SEP after the shortening of the spinal cord. A significant prolongation was observed in all the experimental groups at 5 min post-shortening. In Group B and $C$, the changes returned to normal level in $6 \mathrm{~h}$ after surgery. ${ }^{*} P<0.05$ indicates that the values were significantly different from those before the operation. 
level at $6 \mathrm{~h}$ after the shortening $(P=0.033)$, and that in group $\mathrm{D}$ fell under the baseline level at $6 \mathrm{~h}$ after the shortening $(P=0.041)$. Different from that in the above three groups, the SCBF of Group E decreased immediately at $5 \mathrm{~min}$ after the shortening $(P=0.017)$, and presented a progressive fall in the next few hours. (Figure 4, Table 3 ).

Results of HE staining and transmission electron microscope Punctate hemorrhage was seen in Group $\mathrm{C}$ while splinter hemorrhage was observed in Group D and E, suggesting that hemorrhage became

Table 1 Mean ( \pm s.e.m.) latency of SEP before and after the shortening of the spinal cord (ms, $n=6)$

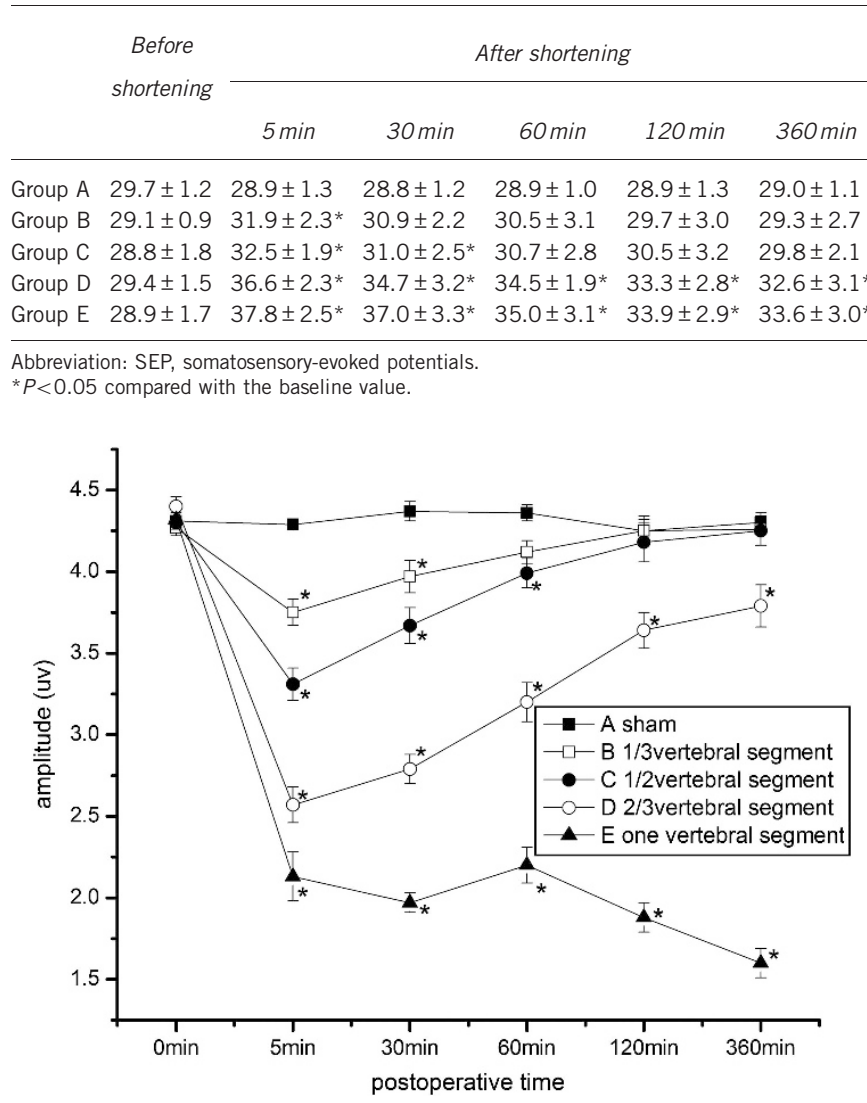

Figure 3 Changes of the amplitude of SEP after the shortening of the spinal cord. Amplitude decreased significantly at $5 \mathrm{~min}$ post-shortening in all the experimental groups, and then showed a tendency to recover. In Group D and $E$, the amplitudes were still significantly lower $6 \mathrm{~h}$ after surgery. ${ }^{*} P<0.05$ indicates that the values were significantly different from those before the operation. more serious when the shortening was longer. The hemorrhages were usually found in gray matter, especially in the junction of grey matter and white matter (Figure 5). The main changes of the white matter were swelling of axonal (observed in Group C), different degrees of myelin damage (observed in Group D and E) and even demyelination (observed in Group E) (Figures 5 and 6).

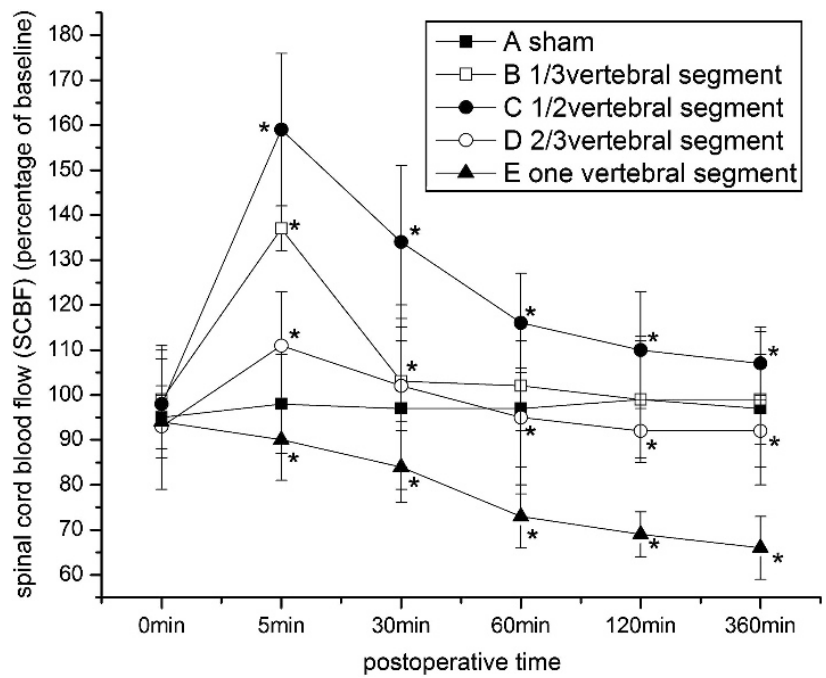

Figure 4 Changes of SCBF after the shortening of the spinal cord. SCBF of Group $B, C$ and $D$ increased temporarily at $5 \mathrm{~min}$ after the shortening. SCBF in Group $E$ decreased immediately at $5 \mathrm{~min}$ post operation and fell continuously. ${ }^{*} P<0.05$ indicates that the values were significantly different from the baseline value.

Table 3 Mean SCBF ( \pm s.e.m.) over time before and after the shortening of the spinal cord expressed as a percentage relative to the baseline level. $(n=6)$

\begin{tabular}{lrrrrrr}
\hline \multirow{6}{*}{$\begin{array}{c}\text { Before } \\
\text { shortening }\end{array}$} & \multicolumn{5}{c}{ After shortening } \\
\cline { 3 - 7 } & & $5 \mathrm{~min}$ & $30 \mathrm{~min}$ & $60 \mathrm{~min}$ & $120 \mathrm{~min}$ & $360 \mathrm{~min}$ \\
\hline Group A & $95 \pm 16^{*}$ & $98 \pm 11$ & $97 \pm 18$ & $97 \pm 19$ & $99 \pm 14$ & $97 \pm 17$ \\
Group B & $99 \pm 11$ & $137 \pm 5^{*}$ & $103 \pm 9^{*}$ & $102 \pm 10$ & $99 \pm 13$ & $99 \pm 10$ \\
Group C & $98 \pm 10$ & $159 \pm 17^{*}$ & $134 \pm 17^{*}$ & $116 \pm 11^{*}$ & $110 \pm 13^{*}$ & $107 \pm 8^{*}$ \\
Group D & $93 \pm 7^{*}$ & $111 \pm 12^{*}$ & $102 \pm 18$ & $95 \pm 11^{*}$ & $92 \pm 7^{*}$ & $92 \pm 8^{*}$ \\
Group E & $94 \pm 8^{*}$ & $90 \pm 9^{*}$ & $84 \pm 8^{*}$ & $73 \pm 7^{*}$ & $69 \pm 5^{*}$ & $66 \pm 7^{*}$ \\
\hline Aboyyyy
\end{tabular}

Abbreviation: SCBF, spinal cord blood flow.

${ }^{*} P<0.05$ compared with the baseline value.

Table 2 Mean ( \pm s.e.m.) amplitude of SEP before and after the shortening of the spinal cord $(\mu v, n=6)$

After shortening

\begin{tabular}{|c|c|c|c|c|c|c|}
\hline & & $5 \mathrm{~min}$ & $30 \mathrm{~min}$ & $60 \mathrm{~min}$ & $120 \mathrm{~min}$ & $360 \mathrm{~min}$ \\
\hline Group A & $4.31 \pm 0.02$ & $4.29 \pm 0.03$ & $4.37 \pm 0.06$ & $4.36 \pm 0.05$ & $4.25 \pm 0.07$ & $4.30 \pm 0.06$ \\
\hline Group B & $4.27 \pm 0.05$ & $3.75 \pm 0.08^{*}$ & $3.97 \pm 0.10$ * & $4.12 \pm 0.07$ & $4.25 \pm 0.09$ & $4.26 \pm 0.10$ \\
\hline Group C & $4.30 \pm 0.04$ & $3.31 \pm 0.10^{*}$ & $3.67 \pm 0.11^{*}$ & $3.99 \pm 0.09 *$ & $4.18 \pm 0.12$ & $4.25 \pm 0.09$ \\
\hline Group D & $4.40 \pm 0.06$ & $2.57 \pm 0.11^{*}$ & $2.79 \pm 0.09 *$ & $3.20 \pm 0.12^{*}$ & $3.64 \pm 0.11^{*}$ & $3.79 \pm 0.13 *$ \\
\hline Group E & $4.32 \pm 0.04$ & $2.13 \pm 0.15^{*}$ & $1.97 \pm 0.06^{*}$ & $2.20 \pm 0.11^{*}$ & $1.88 \pm 0.09 *$ & $1.60 \pm 0.09 *$ \\
\hline
\end{tabular}

Abbreviation: SEP, somatosensory-evoked potentials.

${ }^{*} P<0.05$ compared with the baseline value. 

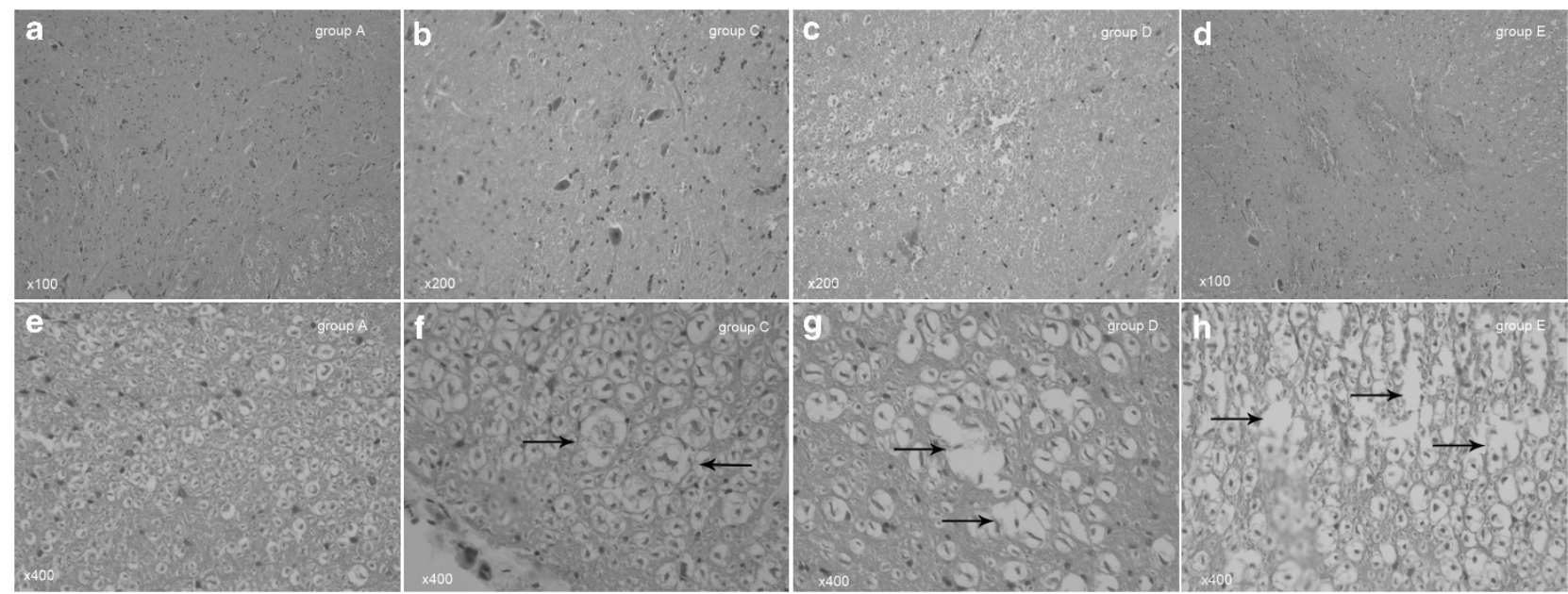

Figure 5 Histopathologic changes of the shortened spinal cord with HE stain. Grey (a) and white (e) matter preserved well in a sham dog. Hemorrhage in grey matter worsened (punctate hemorrhage in $\mathbf{b}$ and $\mathbf{c}$, and splinter hemorrhage in $\mathbf{d}$ ), and changes of white matter deteriorated (axons swelling in $\mathbf{f}$ and $\mathbf{g}$, and myelin sheats split off in $\mathbf{h}$, black arrow).
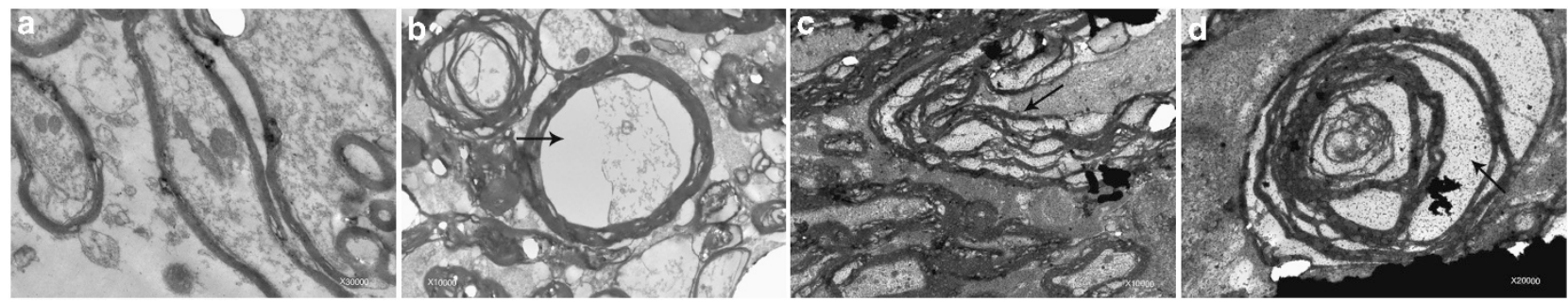

Figure 6 Histopathologic changes of the shortened spinal cord under electron microscope. Axons were almost complete in sham control (a). Periaxonal space and edema occurred when the shortening was of half of the vertebral height (b, black arrow). Myelin breakdown (black arrow) was observed when the shortening was of two-thirds (c) and even total of a vertebral height (d).

\section{DISCUSSION}

The present study built canine models of acute shortening of the spinal cord after spondylectomy, and investigated the variations of SEP and SCBF in different stages during the shortening so as to determine the safe shortening range that would not induce SCI. The microstructure and ultrastructure of the spinal cord were observed to verify the results. These findings would be helpful for further investigations of the mechanisms of injuries induced by spinal cord shortening and preventing SCI in the process of the surgery.

In the previous studies, ${ }^{6,7}$ no instrumentation was performed on the osteotomy surfaces of the animal models and the bone surfaces were not contacted. In our experiment, part of the vertebral body was resected when the spinal shortening was $<$ one vertebral height, so that the bone-to-bone contact of the adjacent vertebral bodies was achieved and the human surgery was better simulated in animal models. The maximum amount of the spinal cord shortening that would not induce SCI was determined as half of a vertebral segment, which was much $<104.2 \%$ of one vertebral body height reported by Modi. ${ }^{7}$ The reason of this difference might be that two laminaes were removed in Modi's study when the shortening was half of a vertebral column height, while only half of laminae was removed in ours. Similarly, only part of the laminae was resected when the shortening was $<$ one vertebral height, which was $<$ the one segment resected in Kawahara's study, so that incomplete SCI occurred when the shortening reached two-thirds of a vertebral segment in our animals. These findings physiologically support the conclusion of Alemdaroğlu that resection of even a half more laminae might reduce the compression of the spinal cord. ${ }^{8}$ On the other hand, we hypothesize that increasing the length of vertebral resected might also prevent excessive shortening of the spinal cord, which needs further research to corroborate. And, of course, the selection of different experimental animals might also be contributory to the different result.

SEP is the most commonly used method of neurophysiological monitoring of spinal function,. An amplitude loss of SEP of $>50 \%$ or an increase of latency over $10 \%$ is generally considered meaningful. ${ }^{13}$ However, some researchers argue that the change of amplitude alone is not adequate to be the criterion for SCI, and the duration of the change should also be taken into account. ${ }^{13}$ In the studies of Kawahara ${ }^{6}$ and Modi, ${ }^{7}$ the fluctuation of SEP amplitude alone was set as the signal of SCI. Whereas, in our study, the amplitude and latency were examined quantitatively and over time, which would lead to a more sensible and reliable judgement of SCI, especially of incomplete injury. Additionally, it was also found that amplitude was more sensitive than latency to SCI.

The laser doppler flowmetry technique is an easy and non-invasive method that allows continuous blood flow recordings in a restricted tissue. ${ }^{12,14,15}$ SCBF has an important role in SCI, especially in secondary injury. ${ }^{16}$ The previous studies did not consider the continuous SCBF changes over time, which might be attributable to short observation time (30 min in Modi's study) or limitations of the method (spinal cord shortening at different levels was performed on one animal). It was found in this experiment that SCBF increased immediately during the first $5 \mathrm{~min}$ after the spinal cord was shortened 
within two-thirds of the vertebral segment. What is worth noticing is that it showed a tendency to return to the baseline level following the temporary increase. This reversible change may be related to the autoregulatory ability of the spinal cord. Acute SCI consists of the primary injury, as well as the secondary injury which includes inflammation, free radicals, and so on. ${ }^{17}$ These physiological and pathological events have a close relationship with SCBF. When the amount of the shortening of the spinal cord reaches a whole vertebral segment height, the primary injury may go beyond the autoregulatory ability of the spinal cord. Subsequently, the SCBF decreases sharply and the secondary injury mechanisms may aggravate the primary mechanical injury. ${ }^{18}$

The successional variation of SCBF after the spinal cord shortening found in our study can provide important information for fundamental and clinical research. First, it provides basic information for understanding the role of SCBF response in the pathophysiology of spinal cord shortening induced SCI, which is important for further investigation of the mechanism of injury. For example, series of pathophysiological changes may be triggered by the rapid increase of SCBF in the early stage of spinal cord shortening. Secondly, it is critical for the design and implementation of therapeutic or prophylactic treatment strategies, and pharmacological interventions. For instance, some treatments that raise or lower SCBF in the early period might prevent or alleviate SCI. The histopathologic findings in the current study warn us that intramedullary hemorrhage and large-area myelin breakdown may happen when the amount of shortening reaches the height of one vertebral segment. Combining our findings with those of Kawahara and Modi, we have drawn the conclusion that increasing the length in laminectomy can expand the safe range of spinal cord shortening. The data obtained in this study can provide evidence for clinical surgery.

The measurement of SEP and SCBF can be affected by many factors, among which the paramount one is the effect of anesthetics (especially the halogenated inhalational agents). ${ }^{19}$ In our experiment, the inhalational agents were not used in order to minimize the influences of anesthetics. The core body temperature was controlled using a heating blanket. Mean blood pressure, another critical influential factor, ${ }^{20}$ should also be controlled during the measurement. In the present study, the continuous bleeding from the osteotomy was controlled by electric coagulation and bone wax and the systolic blood pressure was maintained between 110 and $130 \mathrm{~mm} \mathrm{Hg}$ by injection of $6 \%$ dextran. Even though, the blood pressure could not be maintained for long time and thus some long-term data were unavailable, such as hindlimb function recovery and long-term histopathology. Another limitation of this study is that the data and conclusions obtained were all based on normal spinal cords; however, in many cases, the spinal cords get injured because of severe spine malformation and these abnormal spinal cords may represent different responses to the shortening, for instance, being more vulnerable to injury.

In conclusion, SEP and SCBF are not stable after the shortening of the spinal cord, but change with time. It is safe to shorten the spinal cord within half of a vertebral height in thoraco-lumbar spine. The shortening between half and two-thirds of a vertebral segment can lead to incomplete SCI. These results have by now been limited in animal models, but the presentation of SEP, SCBF and pathophysiological changes after different amount of shortening of the spinal cord in animal models may be consistent with those in human. Therefore, further studies with animal models are necessary, especially on mechanisms of secondary SCI and therapeutic or prophylactic treatment strategies.

\section{DATA ARCHIVING}

There were no data to deposit.

\section{CONFLICT OF INTEREST}

The authors declare no conflict of interest.

\section{ACKNOWLEDGEMENTS}

This work was supported by Technology Development Foundation of Shaanxi Province (No.2009K-01)

1 Lehmer SM, Keppler L, Buscup RS, Enker P, Miller SD, Steffee AD. Posterior transvertebral osteotomy for adult thoracolumbar kyphosis. Spine (Phila Pa 1976) 1994: 19: 2060-2067.

2 Lenke LG, Sides BA, Koester LA, Hensley M, Blanke KM. Vertebral column resection for the treatment of severe spinal deformity. Clin Orthop Relat Res 2010; 468 687-699.

3 Manabe S, Tateish A, Abe M, Ohno T. Surgical treatment of metastatic tumor of the spine. Spine (Phila Pa 1976) 1989; 14: 41-47.

4 Padrals JG. Spinal shortening in scoliosis surgery: a case with transitory paraplegia. Spine (Phila Pa 1976) 1996; 21: 2515-2519.

5 Suk SI, Kim JH, Kim WJ, Lee SM, Chung ER, Nah KH. Posterior vertebral column resection for sever spinal deformities. Spine (Phila Pa 1976) 2002; 27: 2374-2382.

6 Kawahara N, Tomita K, Kobayashi T, Abdel-Wanis ME, Murakami H, Akamaru T. Influence of acute shortening on the spinal cord: an experimental study. Spine (Phila Pa 1976) 2005; 30: 613-620.

7 Modi HN, Suh SW, Hong JY, Yang JH. The effects of spinal cord injury induced by shortening on motor evoked potentials and spinal cord blood flow, an experimental study in swine. J Bone Joint Surg AM 2011; 93: 1781-1789.

8 Alemdaroglu KB, Atlihan D, Cimen O, Kilinc CY, IItar S. Morphometric effects of acute shortening of the spine: the kinking and the sliding of the cord, response of the spinal nerves. Eur Spine J 2007; 16: 1451-1457.

9 Jasper $\mathrm{HH}$. The ten-twenty electrode system of the International Federation. Electroencephalogr Clin Neuro 1958; 10: 371-375.

10 Oostrom H. Stience PJ, Doornenbal A, Hellebrekers LJ. Nociception-related somatosensory evoked potentials in awake dogs recorded after intra epidermal electrica stimulation. Euro J Pain 2009; 13: 154-160.

11 Kurosawa M, Toda $\mathrm{H}$, Watanabe $\mathrm{O}$, Budgell B. Contribution of supraspinal and spina structures to the responses of dorsal spinal cord blood flow to innocuous cutaneous brushing in rats. Auton Neurosci Basic Clin 2007; 136: 96-99.

12 Rane K, Segerdahl M, Karlsten R. Intrathecal adenosine increases spinal cord blood flow in the rat: measurements with the laser-doppler flowmetry technique. Act Anaesth Scand 2004; 48: 1249-1255.

13 Macri S, De Monte A, Greggi T, Parisini P, Zanoni A, Merlini L. Intra-operative spinal cord monitoring in orthopaedics. Spinal Cord 2000; 38: 133-139.

14 Harakawa I, Yano T, Sakurai T, Nishikimi N, Nimura Y. Measurement of spinal cord blood flow by an inhalation method and intraarterial injection of hydrogen gas. J Vasc Surg 1997; 26: 623-628.

15 Frerichs KU, Feuerstein GZ. Laser-Doppler-flowmetry: a review of its application for measuring cerebral and spinal cord blood flow. Mol Chem Neuropathol 1990; 12 55-70.

16 Martirosyan NL, Feuerstein JS, Theodore N, Cavalcanti DD, Spetzler RF, Preul MC. Blood supply and vascular reactivity of the spinal cord under normal and pathological conditions A review. J Neurosurg Spine 2011; 15: 238-251.

17 Oyinbo CA. Secondary injury mechanisms in traumatic spinal cord injury: a nugget of this multiply cascade. Acta Neurobiol Exp 2011; 71: 281-299.

18 Mautes AEM, Weinzier MR, Donovan F, Noble L. Vascular events after spinal cord injury: contribution to secondary pathogenesis. Phys Ther 2000; 80: 673-687.

19 Sloan TB. Anesthetic effects on electrophysiologic recordings. J Cl Neuroph 1998; 15 217-226.

20 Seyal M, Mull B. Mechanisms of signal change during intraoperative somatosensory evoked potential monitoring of spinal cord. J Cl Neuroph 2002; 19: 409-415. 